\title{
Mielopatia cervical secundária a gota: Relato de caso*
}

\section{Cervical Myelopathy Secondary to Gout: Case Report}

\author{
Thiago Dantas Matos ${ }^{10}$ Kelsen de Oliveira Teixeira ${ }^{10}$ Rodrigo Barra Caiado Fleury ${ }^{10}$ \\ Herton Rodrigo Tavares Costa ${ }^{1 \odot}$ Joel Del Bel Pádua ${ }^{2}$ Helton Luiz Aparecido Defino ${ }^{1 \odot}$ \\ 1 Departamento de Ortopedia e Traumatologia, Universidade de São Paulo, \\ Faculdade de Medicina de Ribeirão Preto, Ribeirão Preto, SP, Brasil \\ 2 Departamento de Patologia, Universidade de São Paulo, Faculdade \\ de Medicina de Ribeirão Preto, Ribeirão Preto, SP, Brasil \\ Endereço para correspondência Thiago Dantas Matos, MD, \\ Departamento de Ortopedia e Traumatologia, Universidade de São \\ Paulo, Faculdade de Medicina de Ribeirão Preto, Avenida \\ Bandeirantes, 3900, Monte Alegre, Ribeirão Preto, SP, 14049900, \\ Rev Bras Ortop 2020;55(6):796-799. \\ Brasil (e-mail: tdmatos@usp.br).
}

\section{Resumo \\ Palavras-chave \\ - gota \\ - compressão da medula espinal \\ - paraparesia}

A gota é uma artropatia cristalina frequente na população; entretanto, a espondiloartropatia gotosa, também chamada de gota axial, é incomum. O presente relato de caso apresenta um caso raro de mielopatia cervical secundária a gota axial. Uma paciente de 50 anos de idade, sem patologias prévias, apresentou quadro de perda de força, alteração de sensibilidade e liberação piramidal há 2 anos. A tomografia computadorizada evidenciou imagem lítica no processo espinhoso de C7, e sinais de mielopatia com mielomalácia foram observados na ressonância magnética da coluna cervical. Após o procedimento cirúrgico e biópsia do material, o diagnóstico foi de gota, e o tratamento para a patologia foi iniciado, com melhora completa do quadro. O diagnóstico de gota axial deve ser incluído no espectro do diagnóstico diferencial das doenças que acometem a coluna vertebral. Apesar de a espondiloartrite gotosa ser incomum, há uma ocorrência subestimada devido a não investigação dos casos. O diagnóstico precoce e tratamento da patologia pode evitar que pacientes apresentem complicações da doença, como a relatada no presente estudo.

Gout is a crystalline arthropathy frequent in the population, but gouty spondyloarthropathy, also called axial gout, is uncommon. The current case report presents a rare case of cervical myelopathy secondary to axial gout. A 50-year-old female patient, without previous pathologies, presented with loss of strength, altered sensitivity, and pyramidal release for 2 years. The computed tomography showed a lytic image in the spinous process of $C 7$, and signs of myelopathy with myelomalacia on magnetic resonance imaging of the cervical spine. After the surgical procedure and biopsy of the material, the diagnosis was gout, and treatment for the pathology was started, with complete improvement of the condition. The diagnosis of axial gout should be included in the spectrum of the differential diagnosis of diseases that affect the spine. Although gouty spondyloarthritis (or spondylitis) is uncommon, there is an underestimated occurrence due to the lack of investigation of the cases. The early diagnosis and treatment of the pathology can prevent patients from presenting complications of the disease, as reported in the present study.

\footnotetext{
* Trabalho desenvolvido no Departamento de Ortopedia e Traumatologia da Faculdade de Medicina de Ribeirão Preto, Universidade de São Paulo, Ribeirão Preto, SP, Brasil.
}

recebido

12 de Agosto de 2019 aceito

12 de Dezembro de 2019
DOI https://doi.org/

10.1055/s-0040-1708514. ISSN 0102-3616.
Copyright $\odot 2020$ by Sociedade Brasileira License terms de Ortopedia e Traumatologia. Published by Thieme Revinter Publicações Ltda, Rio de Janeiro, Brazil 


\section{Introdução}

A gota é a artropatia cristalina mais frequente, com prevalência global de $0,08 \%$, e acomete prefrencialmente o sexo masculino após a quinta década de vida. No entanto, a espondiloartropatia gotosa (EG) é incomum e mimetiza uma variedade de doenças que acometem a coluna vertebral, representando desafio para o seu diagnóstico na prática clínica. ${ }^{1-3}$

A EG, também denominada de de gota axial ou da coluna vertebral, foi descrita em 1950 por Kersley et al., ${ }^{4}$ e Kosoff et al. (1953) foram os pioneiros no relato da mielopatia por gota. ${ }^{5} \mathrm{~A}$ gota axial é uma manifestação incomum dessa doença, com amplo espectro de manifestação clínica da doença, variando desde a forma assintomática até quadros graves de compressão das estruturas nervosas com paraplegia ou tetraparesia. ${ }^{1} \mathrm{O}$ objetivo desse relato de caso clínico é apresentar o acometimento da coluna vertebral na gota acompanhado de informações acerca dessa manifestação incomum da doença, que deve ser considerada no diagnóstico diferencial.

O estudo foi aprovado pela comissão institucional de ética em pesquisa sob o número CAAE 19635119.8.0000.5440.

\section{Relato do Caso}

Uma paciente de 50 anos, do sexo feminino, foi admitida com queixa de dor cervical com irradiação para membros superiores e parestesia no dermátomo de C6 e C7. Relatava quedas frequentes e dificuldade para deambular há 2 anos. 0 exame físico revelou fraqueza bilateral dos membros superiores, com força muscular grau quatro de acordo com a escala do Medical Research Council (MRC), e com maior acometimento dos miótomos C6 e C7. Os sinais de Hoffmann e Babinski eram positivos em ambos os lados.

Os exames laboratoriais da rotina pré-operatória não apresentavam alteração e nenhuma hipótese diagnóstica pôde ser realizada para esclarecimento das lesões líticas dos elementos posteriores de C7 (- Figura 1). O exame de ressonância magnética da coluna cervical apresentava estenose do canal medular, principalmente nos níveis de C6 a C7 (-Figura 2).

A abordagem posterior da coluna cervical foi realizada para permitir a estabilização e descompressão posterior da coluna cervical e acesso aos elementos posteriores de $\mathrm{C} 7$ para a obtenção do material para o exame anatomopatológico. Foram realizados a artrodese e a fixação posterior de C4 a T1 associados com a laminectomia de $\mathrm{C} 5$ a $\mathrm{C} 7$. O processo espinhoso e o arco de $\mathrm{C7}$, que apresentavam a lesão lítica, foram encaminhados para exame anatomopatológico para investigação diagnóstica (-Figura 3).

No período pós-operatório a paciente apresentou melhora significativa da dor cervical e sintomas neurológicos, com boa evolução da ferida cirúrgica, sem complicações gerais ou específicas relacionadas com o procedimento cirúrgico realizado.

Ao exame macroscópico, a lesão de C7 era composta por tecido acastanhado e macio, e os cortes histológicos corados por hematoxilina e eosina (HE) mostraram que o tecido ósseo não apresentava alterações histológicas. Foram ainda observados uma porção adjacente de tecido sinovial com hiperplasia vilosa, infiltrado inflamatório linfocítico leve e depósitos de

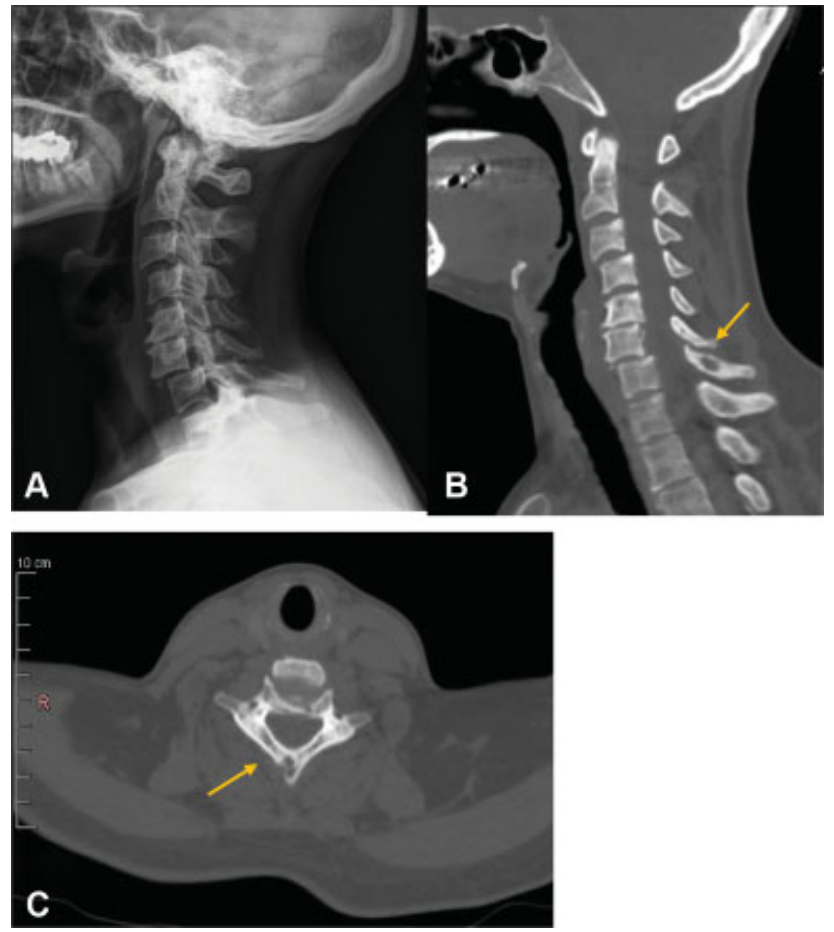

Fig. 1 (A) Radiografia em perfil da coluna cervical evidenciando alterações degenerativas dos segmentos vertebrais e deslocamento anterior de C6. (B) Tomografia computadorizada evidenciando lesão lítica do processo espinhoso C7 no corte sagital (seta amarela). (C) Corte axial da tomografia computadorizada evidenciando múltiplas lesões líticas nos elementos posteriores de C7. (seta amarela).
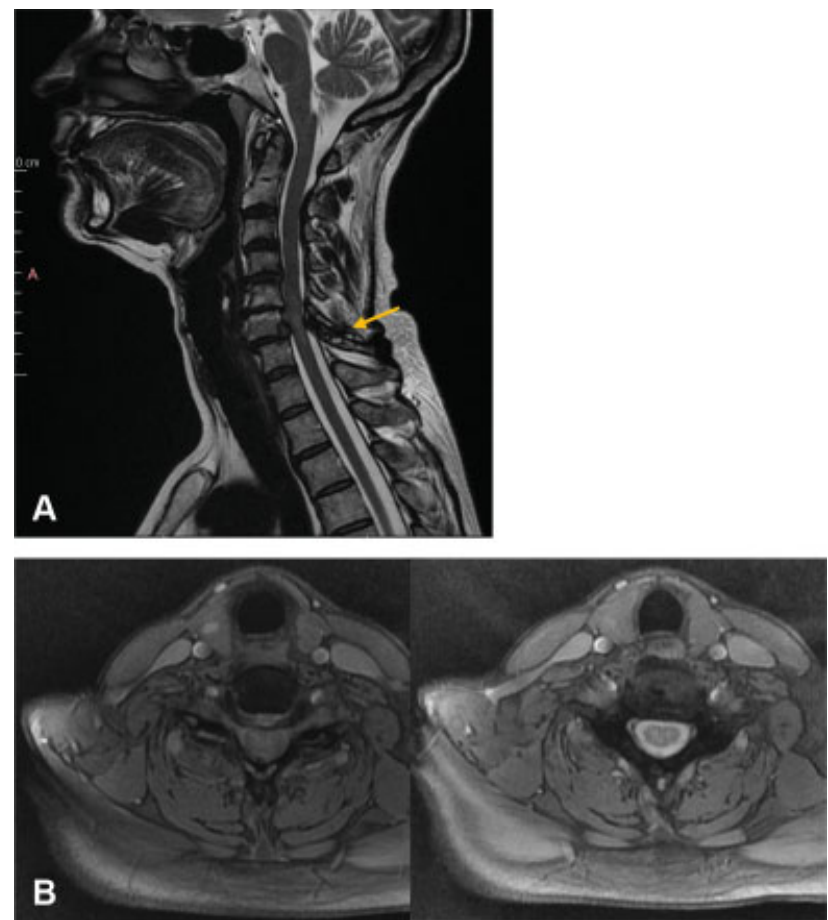

Fig. 2 (A) Ressonância magnética evidenciando estenose cervical C6/ $C 7$, lesões líticas com área de tecido inflamatório no processo espinhoso e lâmina de C7. (B) Corte axial da ressonância magnética ponderada em T2 evidenciando a estenose do canal medular. 


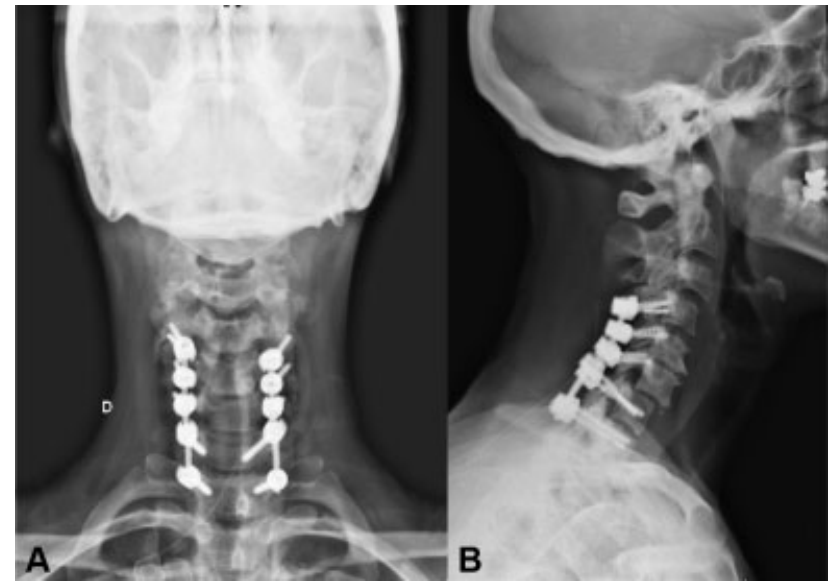

Fig. 3 Radiografias do pós-operatório, (A) Anteroposterior, (B) Perfil.

urato circundado por histiócitos e céluas gigantes de corpo estranho, compatíveis com o diagnóstico de sinovite gotosa (-Figura 4).

Após o resultado do exame anatomopatológico indicando o diagnóstico de gota, foram realizados os exames complementares específicos para essa doença, e o tratamento foi iniciado com a administração de Alupurinol, $300 \mathrm{mg} /$ dia, associada com a dieta apropriada. A paciente apresentou boa evolução dos sintomas álgicos relacionados com a gota, e a evolução do resultado dos exames realizados durante o seguimento mostrou queda dos valores do ácido úrico sérico (-Tabela 1).

\section{Discussão}

A gota axial é uma manifestação incomum da artrite gotosa, mas os relatos de casos clínicos ultrapassam a primeira centena, indicando que a sua ocorrência possa ser subestimada. ${ }^{1} \mathrm{O}$ diagnóstico de gota axial da paciente que estamos relatando ocorreu devido aos sintomas neurológicos que ela apresentava e à realização do exame anatomopatológico, pois não existiam evidências clínicas da doença. A gota axial pode ser mais frequente do que consideramos, e somente os
Tabela 1 Valores dos exames laboratoriais durante seguimento ambulatorial

\begin{tabular}{|l|l|l|l|l|}
\hline & $\mathbf{1}^{\circ}$ coleta & $\mathbf{2}^{\circ}$ coleta & $3^{\circ}$ coleta & $4^{\circ}$ coleta \\
\hline $\begin{array}{l}\text { Ácido } \\
\text { úrico } \\
\text { sérico }\end{array}$ & $15,5 \mathrm{mg} / \mathrm{dL}$ & $8,2 \mathrm{mg} / \mathrm{dL}$ & $9,7 \mathrm{mg} / \mathrm{dL}$ & $3,2 \mathrm{mg} / \mathrm{dL}$ \\
\hline Ureia & & $35,7 \mathrm{mg} / \mathrm{dL}$ & $35,8 \mathrm{mg} / \mathrm{dL}$ & $31,4 \mathrm{mg} / \mathrm{dL}$ \\
\hline Creatinina & $1,0 \mathrm{mg} / \mathrm{dL}$ & $1,1 \mathrm{mg} / \mathrm{dL}$ & $1,2 \mathrm{mg} / \mathrm{dL}$ & $1,0 \mathrm{mg} / \mathrm{dL}$ \\
\hline TGO & & & $23 \mathrm{U} / \mathrm{L}$ & $23 \mathrm{U} / \mathrm{L}$ \\
\hline TGP & & & $18 \mathrm{U} / \mathrm{L}$ & $19 \mathrm{U} / \mathrm{L}$ \\
\hline
\end{tabular}

Abbreviations: TGO, transaminase glutâmico-oxalacética; TGP, transaminase glutâmico-pirúvica.

pacientes com sintomas têm sido considerados nos relatos de casos. ${ }^{1,6,7}$ Noventa e oito por cento dos pacientes analisados em um artigo de revisão de gota axial apresentavam sintomas neurológicos, ${ }^{1}$ e o estudo radiológico prospectivo de pacientes com gota evidenciou lesões erosivas na coluna vertebral em pacientes assintomáticos. ${ }^{8}$

Os pacientes do sexo masculino e com a média de idade ao redor dos 60 anos de idade têm sido os mais acometidos pela gota axial, ${ }^{1}$ contrastando com a paciente que relatamos. No entanto, a paciente apresentava aumento do ácido úrico, que é relatado em cerca de $69,6 \%$ dos pacientes com gota. ${ }^{1}$

Todos os segmentos da coluna vertebral têm sido acometidos pela gota axial, sendo a coluna lombar a mais frequente (58,4\%). A localização cervical, que foi a observada em nossa paciente, corresponde ao segundo segmento em frequência, e foi relatado em $24,8 \%$ dos pacientes. Qualquer estrutura da coluna vertebral pode ser afetada, bem como os tecidos adjacentes. $^{1}$

Os sintomas neurológicos observados nessa paciente estão de acordo com a maioria dos relatos, nos quais $77,9 \%$ dos pacientes apresentavam sintomas neurológicos, que estavam associados com dor no segmento acometido da coluna vertebral. A radiculopatia é o sintoma mais frequente, e é relatada em $34,5 \%$ dos pacientes, seguida de claudicação neurogênica $(20,4 \%)^{1}$

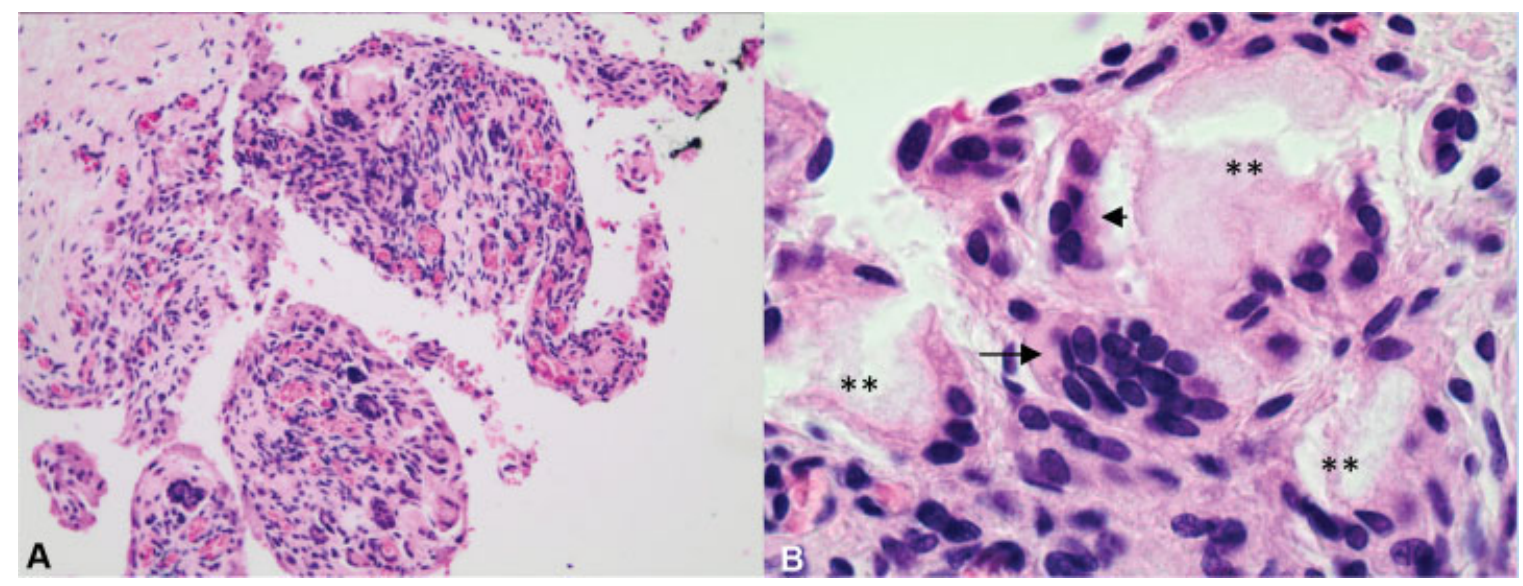

Fig. 4 Corte histológico corado com hematoxilina e eosina evidenciando tecido sinovial hiperplásico com infiltrado inflamatório linfo-histiocitário (A). Em maior ampliação (B), notam-se diminutos depósitos de material amorfo $\left({ }^{* *}\right)$ indicativo da presença de urato (os cristais são dissolvidos no processo de fixação e coloração), circundados por macrófagos epitelioides (seta curta) e células gigantes multinucleadas (seta comprida). 
Os achados radiológicos são compatíveis com as alterações da osteoartrite. ${ }^{9}$ Apesar de não esclarecidos, os mecanismos associados com a gota axial têm sido considerados semelhantes ao que ocorre no quadro periférico, no qual o pH, temperatura, trauma, e a degeneração das articulações estão envolvidos no depósito dos cristais. ${ }^{9}$ As alterações radiológicas podem variar desde o edema dos tecidos moles até a erosão do tecido ósseo. ${ }^{9}$ As lesões líticas observadas em nossa paciente, com as características da imagem da tomografia computadorizada e ressonância magnética, são típicas em pacientes com gota, mas não permitem a realização do diagnóstico. Essas lesões permitem apenas a presunção do diagnóstico, que pode ser confirmado apenas com o exame histopatológico, como ocorreu com a nossa paciente. $O$ exame histopatológico é fundamental para o diagnóstico definitivo e exclusão de outras doenças que podem apresentar imagem semelhante. ${ }^{1}$ A obtenção do material para o exame histopatológico pode ser obtida por meio da punção ou pela excisão direta durante o tratamento nos pacientes que apresentam déficit neurológico e necessitam do tratamento cirúrgico. No entanto, a avaliação clínica, laboratorial e radiográfica minuciosa deve preceder essa etapa.

A característica histológica da gota são os depósitos de urato de sódio circundados por variável reação granulomatosa do tipo corpo estranho. Os cristais de urato, que têm cor marrom, são visíveis nos tecidos fixados em álcool. A fixação em formalina combinada ao uso dos corantes aquosos rotineiros, no entanto, dissolve os cristais, restando apenas uma matriz proteica amorfa típica, acinzentada, e com discretas estrias eosinofílicas. ${ }^{10}$

O acometimento vertebral pode ser a manifestação inicial da gota, e deve ser incluído no diagnóstico diferencial, especialmente nos pacientes que apresentam lesão lítica óssea com as características anteriormente descritas.

O caso clínico que relatamos não apresentava história prévia de gota, e o diagnóstico da doença foi realizado pelo exame histopatológico, oriundo do tratamento realizado para a melhora do déficit neurológico, pois a paciente não apresentava sintomas ou diagnóstico prévio de gota.

O diagnóstico de gota deve ser incluído no espectro do diagnóstico diferencial das doenças que acometem a coluna vertebral, e o tratamento da doença deve ser realizado juntamente com o tratamento da doença da coluna vertebral para evitar os problemas ocasionados pela gota. ${ }^{1,4,6,9}$

Conflito de Interesses

Os autores declaram não haver conflito de interesses.

\section{Referências}

1 Hasegawa EM, de Mello FM, Goldenstein-Schainberg C, Fuller R. Gout in the spine. Rev Bras Reumatol 2013;53(03):296-302

2 Toprover M, Krasnokutsky S, Pillinger MH. Gout in the Spine: Imaging, Diagnosis, and Outcomes. Curr Rheumatol Rep 2015;17 (12):70

3 Fernandes ED, Bergamaschi SB, Rodrigues TC, et al. Aspectos relevantes do diagnóstico e seguimento por imagem na gota. Rev Bras Reumatol 2017;57(01):64-72

4 Kersley GD, Mandel L, Jeffrey MR. Gout; an unusual case with softening and subluxation of the first cervical vertebra and splenomegaly. Ann Rheum Dis 1950;9(04):282-304

5 Koskoff YD, Morris LE, Lubic LG. Paraplegia as a complication of gout. J Am Med Assoc 1953;152(01):37-38

$6 \mathrm{Ng} \mathrm{W}$, Sin CH, Wong CH, Chiu WF, Chung OM. Unusual Presentation of Spinal Gout: 2 Cases Report and Literature Review. J Orthop Case Rep 2017;7(06):50-54

7 Volkov A, Rhoiney DL, Claybrooks R. Tophaceous Gout of the Lumbar Spine: Case Report and Review of the Literature. Turk Neurosurg 2015;25(06):954-958

8 Andrés M, Vela P, Volar LC, Avilés Y, Pascual E. Back pain due to lumbar gouty flare-a prospective diagnosis. J Rheumatol 2013;40 (08):1459-1460

9 King JC, Nicholas C. Gouty arthropathy of the lumbar spine: a case report and review of the literature. Spine 1997;22(19):2309-2312

10 Shidham VM, Galindo L, Gupta D, Jhala N, Shidham G. Urate crystals in tissue: A novel staining method for formalin-fixed, paraffin-embedded sections. Lab Med 1998;29:109-113 